\title{
Alignment and physics performance of the Belle II vertex detector
}

\author{
J. Kandra* and T. Bilka et al. on behalf of the Belle II collaboration \\ Charles University \\ E-mail: jakub.kandra@karlov.mff.cuni.cz, \\ bilka@ipnp.troja.mff.cuni.cz
}

The Belle II experiment at the SuperKEKB accelerator will start to take physics data in 2018. One of the major upgrades of the Belle II detector introduces DEPFET pixel sensors in the two innermost layers of its silicon vertex detector, followed by four layers of silicon strip sensors. Excellent performance of the vertex reconstruction is a crucial ingredient in $\mathrm{CP}$ violation measurements and will require reliable and fast alignment procedure and continuous monitoring of the detector performance. To address the possible systematic errors of the procedure, a dedicated study has been performed to identify and evaluate influence of possible random and systematic deformations of the vertex detector on physics observables.

The 15th International Conference on Flavor Physics \& CP Violation

5-9 June 2017

Prague, Czech Republic

\footnotetext{
* Speaker.
} 


\section{Belle II Detector}

Belle II is an experiment under construction at the High Energy Accelerator Research Organization in Tsukuba, Ibaraki prefecture, Japan. Belle II is an upgrade of the Belle experiment which expects to reach integrated luminosity of $\sim 50 \mathrm{ab}^{-1}, 50$ times that of Belle[1]. Belle II will continue and extend the physics program of Belle. The goals of Belle II is to continue the study of $\mathrm{CP}$ violation in B-meson decays and to search for new physics. SuperKEKB accelerator is a next generation high luminosity electron-positron collider with asymmetric energies. It is a double-ring collider with beam energy of $4 \mathrm{GeV}$ for positrons (LER) and $7 \mathrm{GeV}$ for electrons (HER). The accelerator is designed to for instantaneous luminosity of $8 \times 10^{35} \mathrm{~cm}^{-2} \mathrm{~s}^{-1}$. The collider can provide a clean environment for production of $\mathrm{B} \overline{\mathrm{B}}$ pairs, $\tau^{+} \tau^{-}$pairs and $c \bar{c}$ states. The Belle II detector is divided to several parts: the tracking system, the particle identification system, the electromagnetic calorimeter (ECL) and $K_{L}$-muon (KLM) system (Fig. 1).
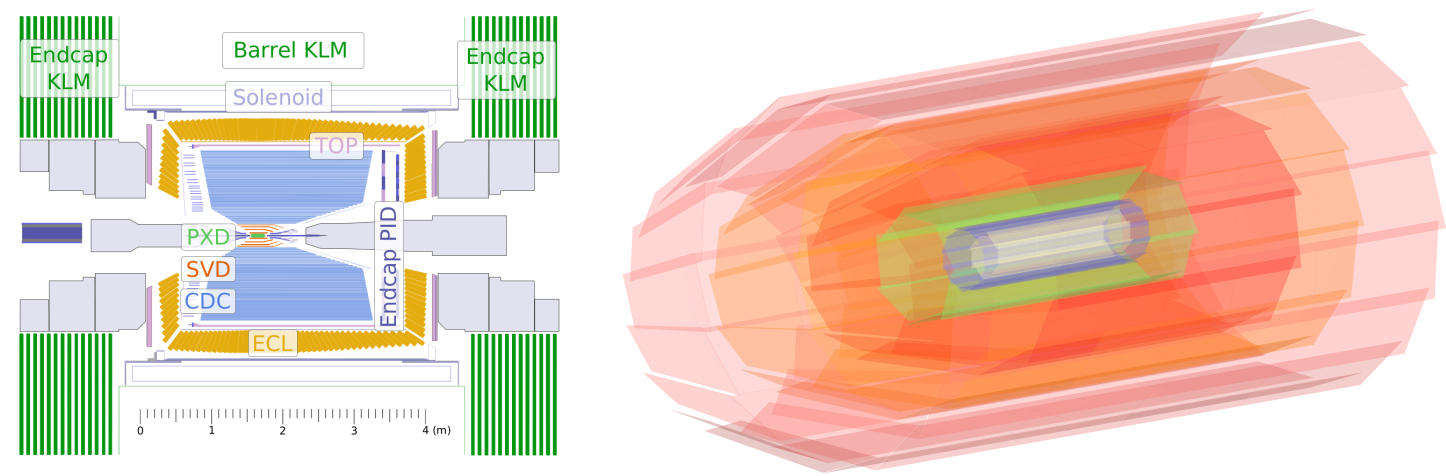

Figure 1: Schematic cross-section of the Belle II detector (left): The PXD (light green), the SVD (orange) and the CDC (blue) compose the tracking system. The TOP (pink) and endcap PID as the ARICH (violet) detector are particle identification detectors. The crystals of the ECL (yellow) surround the PID detectors. The outermost detectors are the barrel and endcap KLM (dark green).

The PXD and SVD in detail (right): Pixel sensors form first (gray) and second (blue) layer. Third (green), fourth (red), fifth (orange) and sixth (pink) layers compose strip detector.

\subsection{Pixel detector}

Pixel sensors form the two innermost layers (Fig. 1). The PXD sensors are based on the DEPFET (DEPleted Field Effect Transistor) technology [2], a semiconductor detector concept combining detection and in-pixel amplification. The thickness of PXD sensors is $75 \mu \mathrm{m}$. Sensitive size of sensor in the first layer is $12.5 \times 44.8 \mathrm{~mm}^{2}$ and in the second layer $12.5 \times 61.44$ $\mathrm{mm}^{2}$. Pixel size of sensors in outer region of the first layer, marked 1.*.1 in Fig. 3, is $50 \times 60 \mu \mathrm{m}^{2}$ and in the central region (sensors 1 . $^{*}$ ) $50 \times 55 \mu \mathrm{m}^{2}$. In the second layer, pixel size for the outer region is $50 \times 85 \mu \mathrm{m}^{2}$ and for the central region it is $50 \times 70 \mu \mathrm{m}^{2}$.

\subsection{Strip detector}

The strip detector (SVD) forms the outer part of the vertex detector. Double-sided silicon strip detectors (DSSD) are used. The basic geometry of the strip, which includes orthogonally implanted 
$\mathrm{n}$ and $\mathrm{p}$ strips on both sides of the detector, provides two coordinate measurements for traversing particles. The detector is composed of 4 layers (Fig. 1). The thickness of SVD sensors is 300 $320 \mu \mathrm{m}$ [3]. Two different sensor shapes are used: rectangular sensors, which will be installed the barrel part, and trapezoidal-shape sensors to cover the forward region. All rectangular silicon sensors are double-sided with the long strips on the p-side, parallel to and facing the beam axis (z). The short n-side strips along $r-\theta$ are located on the sensor face towards the outside. The sensitive area of rectangular sensors in the third layer (3.** in Fig. 3) is $38.4 \times 122.8 \mathrm{~mm}^{2}$ with p-pitch 50 $\mu \mathrm{m}$ at $r-\phi$ side and n-pitch $160 \mu \mathrm{m}$ at $z$ side. The sensitive area of rectangular parallel sensors in layer 4, 5 and 6 is $57.6 \times 122.8 \mathrm{~mm}^{2}$ with p-pitch $75 \mu \mathrm{m}$ at $r-\phi$ side and n-pitch $240 \mu \mathrm{m}$ at $z$ side. The slanted sensors are similar, except that the long strips are not exactly parallel to $\mathrm{z}$. The sensitive area of trapezoidal wedged sensors is $38.4-57.6 \times 122.8 \mathrm{~mm}^{2}$ with p-pitch $50-75$ $\mu \mathrm{m}$ at $r-\phi$ side and n-pitch $240 \mu \mathrm{m}$ at $z$ side. Numbering of VXD layers, ladders and sensors is explained in Fig. 2 and Fig. 3 [4].

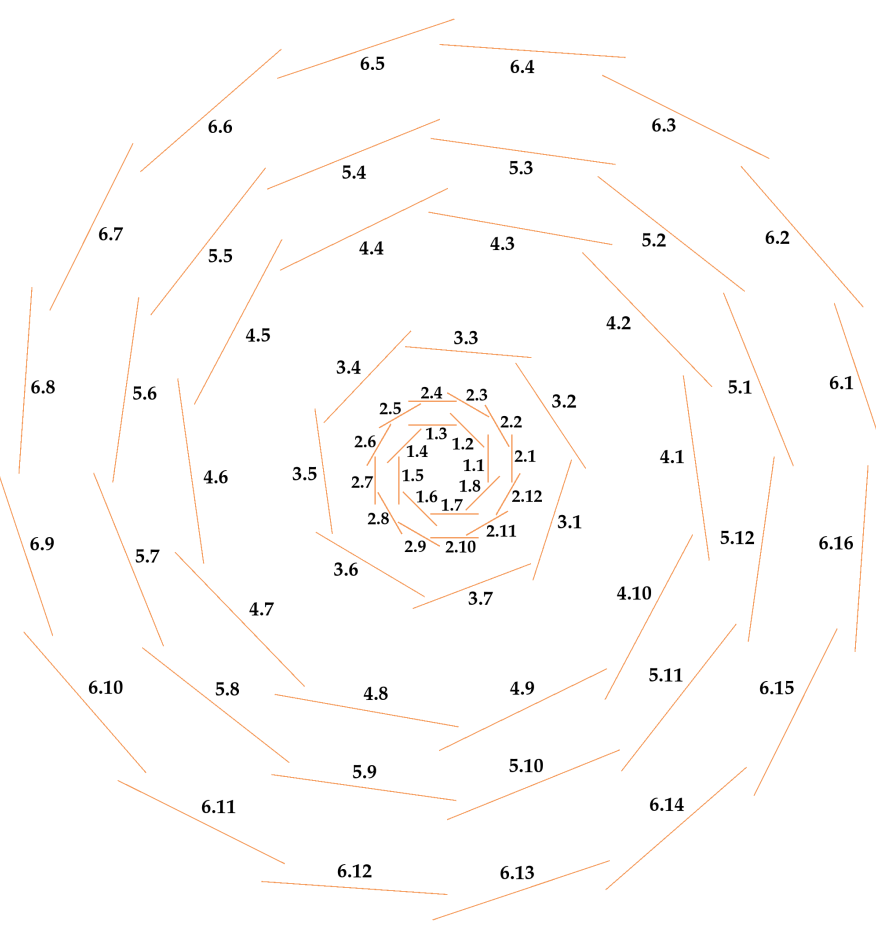

Figure 2: Numbering of VXD ladders: Numbers of sensors are in the format Layer.Ladder

\subsection{Belle II analysis and simulation framework}

The Belle II software system, basf2 (Belle Analysis and Simulation Framework 2), is based on the ideas from the Belle software system and other modern experiments [5]. The most common Linux operating systems are supported. The majority of code is written in $\mathrm{C}++$ while Python scripts are used for run steering. The framework has modular architecture with dynamic library loading. Events are processed sequentially by a chain of modules (path). The selection and arrangement of the modules is defined by the user. Several external libraries are linked to basf 2 to provide the 


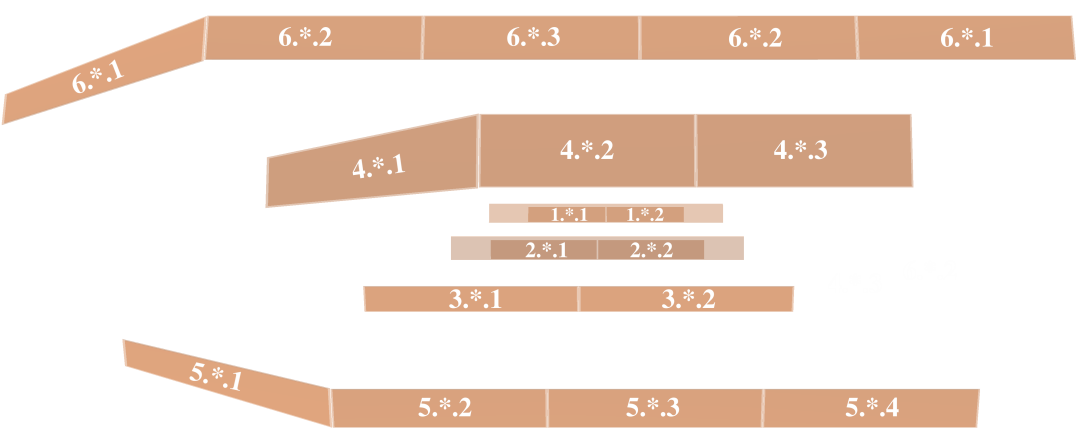

Figure 3: Numbering of VXD sensors: For each layer, the numbering of sensors in its ladders is shown. Numbers of sensors are in the format Layer.Ladder.Sensor

neccessary features. These include ROOT I/O interface needed for processing of events, Geant 4 for full detector simulation or Millepede II and General Broken Lines package as methods for calibration and alignment. The software is able to display events using VGM software.

Simulation of Monte Carlo event samples is done in three steps. The first step involves the generation of events for the various physics studies. The physics of $\mathrm{B}$ decays and $e^{+} e^{-} \rightarrow c \bar{c}$ events are simulated by EvtGen and Pythia. The second step performs the simulation of the interaction of individual particles passing through the detector, described by all its different materials and geometries, and records the particle's energy deposits in sensitive volumes. The simulation is internally done by Geant 4 . After the event is processed, it is augmented with created hits, generated trajectories and created secondary particles. The third step simulates the process of signal generation, electronics effects and final detector hit creation. The digitization software performs for each detector component a detailed simulation of in-detector physics processes together with electronic effects, taking into account the detector geometry and magnetic field information accessible via the geometry interface.

\begin{tabular}{ccccccc} 
& $\begin{array}{c}\text { Layer } \\
\text { Radius } \\
{[\mathrm{mm}]}\end{array}$ & Ladders & $\begin{array}{c}\text { Sensors per } \\
\text { ladder }\end{array}$ & Sensors & $\begin{array}{c}\text { Alignment } \\
\text { parameters }\end{array}$ \\
\hline PXD & 1 & 14 & 8 & 2 & 16 & 96 \\
PXD & 2 & 22 & 12 & 2 & 24 & 144 \\
SVD & 3 & 39 & 7 & 2 & 14 & 84 \\
SVD & 4 & 80 & 10 & 3 & 30 & 180 \\
SVD & 5 & 104 & 12 & 4 & 48 & 280 \\
SVD & 6 & 135 & 16 & 5 & 80 & 480 \\
\hline \hline Total & & 65 & & 212 & 1272
\end{tabular}

Table 1: Number of VXD sensors by layer and ladder 


\section{Alignment of vertex detector}

The vertex detector system provides accurate information on decay vertices. Silicon sensors can measure points on a track very precisely, but to reconstruct the tracks and vertices in space, one needs to know the position of the silicon sensors in space very precisely. Actually, the required precision is much higher than what can be achieved during detector installation. The exact positions of the sensors in space must therefore be estimated from tracks.

The alignment task is to find corrections to nominal positions of sensors. Table 1 shows number of alignment parameters for the Belle II VXD. Over one thousand parameters have to be determined. To estimate these parameters, we use a large set of tracks of several different types and constraints on certain linear combinations of parameters, and use the degrees of freedom remaining after track fits to estimate the alignment parameters.

The normalized tracking residuals are [6]:

$$
z_{i j}=\frac{u_{i j}^{m}-u_{i j}^{p}\left(\boldsymbol{\tau}_{j}, \boldsymbol{a}\right)}{\sigma_{i j}}=\frac{r_{i j}\left(\boldsymbol{\tau}_{j}, \boldsymbol{a}\right)}{\sigma_{i j}},
$$

where $u_{i j}^{m}$ is a recorded measurement of hit $i$ on the track $j, u_{i j}^{p}$ is predicted measurement from track model dependent on track parameters $\boldsymbol{\tau}_{j}$ and alignment parameters $\boldsymbol{a}, \sigma_{i j}$ is the uncertainty of measurement and $r_{i j}$ is a residual. We find optimum track and alignment parameters by minimizing the total $\chi^{2}$ :

$$
\chi^{2}(\boldsymbol{\tau}, \boldsymbol{a})=\sum_{j}^{\text {trackshits }} \sum_{i}^{2} z_{i j}^{2}\left(\boldsymbol{\tau}_{j}, \boldsymbol{a}\right) .
$$

To solve the alignment task, we use the Millepede II algorithm. This algorithm is based on global linear $\chi^{2}$ minimization with constraints. This method relies on linearisation of the normalized residuals $z_{i j}$ in the $\chi^{2}$ function [7]:

$$
\chi^{2}(\boldsymbol{\tau}, \boldsymbol{a})=\sum_{j}^{\text {tracks hits }} \sum_{i}^{2} z_{i j}^{2}\left(\boldsymbol{a}, \boldsymbol{\tau}_{j}\right) \simeq \sum_{j}^{\text {tracks hits }} \sum_{i} \frac{1}{\sigma_{i j}^{2}}\left(r_{i j}\left(\tau_{j}^{0}, \boldsymbol{a}^{0}\right)+\frac{\partial r_{i j}}{\partial \boldsymbol{a}} \delta \boldsymbol{a}+\frac{\partial r_{i j}}{\partial \tau_{j}} \delta \tau_{j}\right)^{2},
$$

where $\boldsymbol{\tau}_{j}^{0}, \boldsymbol{a}^{0}$ are initial alignment and track parameters, $\delta \boldsymbol{\tau}_{j}$ and $\delta \boldsymbol{a}$ are small corrections. Separation of parameters of the tracks and of the alignment allows to simplify the problem by means of block matrix algebra and solve a reduced problem using matrix inversion.

For description of a measurement we use two different coordinates systems: global and local (Fig. 4). For every sensor we use its local frame of measurement, which is defined by $u, v, w$ coordinates. The $w$ is perpendicular to the sensor plane and $u$ and $v$ have directions along the sides of sensor planes with origin at sensor's center, whose position is $\mathbf{r}_{\mathbf{0}}=\left(x_{0}, y_{0}, z_{0}\right)$ in the global system. The vectors are defined as $\mathbf{q}=(u, v, w)$ in the local frame and $\mathbf{r}=(x, y, z)$ in the global system. The transformation from global to local system is [8]:

$$
\mathbf{r}=\mathbf{R}^{T} \Delta \mathbf{R}(\mathbf{q}+\Delta \mathbf{q})+\mathbf{r}_{0}
$$

where $\mathbf{R}$ is a rotation matrix (from global to local frame). The alignment procedure determines correction to initial transformation as an incremental rotation $\Delta \mathbf{R}$ and translation $\Delta \mathbf{q}=(\Delta u, \Delta v, \Delta w)$. The rotation correction $\Delta \mathbf{R}=\mathbf{R}_{\gamma} \mathbf{R}_{\beta} \mathbf{R}_{\alpha}$ is expressed as a product of small rotations $\mathbf{R}_{\alpha}, \mathbf{R}_{\beta}$ and $\mathbf{R}_{\gamma}$ 
by $\Delta \alpha, \Delta \beta, \Delta \gamma$ around the $u$-axis, the new $v$-axis and the new $w$-axis. The translation corrections and rotation angles compose alignment parameters for a single planar sensor $\mathbf{a}=(\Delta u, \Delta v, \Delta w, \Delta \alpha, \Delta \beta, \Delta \gamma)^{T}$.
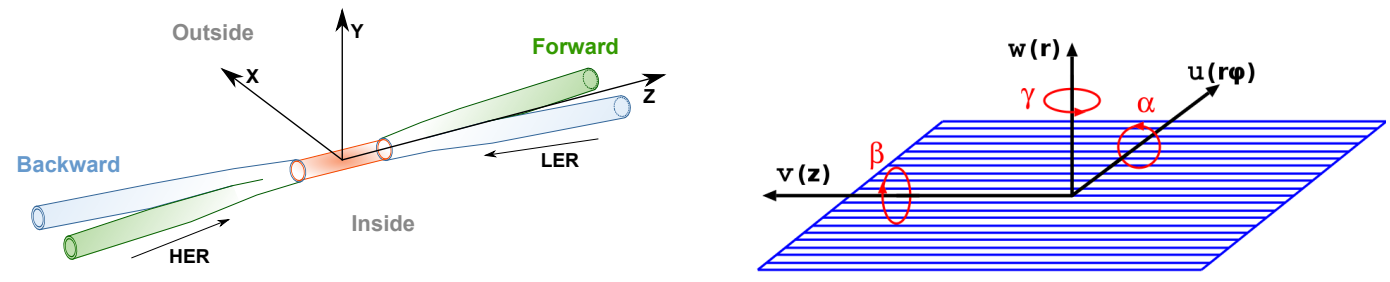

Figure 4: The experiment coordinate system: the global coordinate system of the Belle II detector (left) and a local sensor coordinate system (right)

The trajectory of a charged particle propagating in vacuum with a constant magnetic field is a helix with five parameters. Energy loss in the detector material due to ionization or radiation leads to a reduction of the momentum. Multiple scattering, mainly due to Coulomb interaction with nuclei in the atoms, results in changes in direction and spatial position. The General Broken Lines (GBL) [9] method is a fast global linearised least squares track refit, adding the description of multiple scattering to an initial trajectory. Any material distribution can be described as a thick scatterer, imposing a change of direction and displacement to the particle trajectory. A thick scatterer can be equivalently described by two thin scatterers, which only change the variance of change of direction, at proper positions. The required propagation Jacobians between measurement and scatterrer planes use a locally linearized track model.

\section{Misalignment}

The alignment procedure will be used to determine real positions of silicon sensors during data taking. Initially, the procedure is developed and tested using Monte Carlo simulations. Initial realistic misplacements of silicon sensors are implemented in the third step of the simulation. Two kinds of misalignment are considered: random and systematic misalignment.

\subsection{Random misalignment}

Random misalignment is generated using zero-mean normal distribution for each silicon sensor in rotations and shifts. The standard deviation is used as a measure of the size of the misalignment.

\subsection{Systematic misalignment}

The systematic misalignment is defined as coherent movements of individual sensors (tab. 2), which are poorly controlled within the minimisation procedure because they leave the overall $\chi^{2}$ unaffected. These defects of alignment are called weak modes. If these degrees of freedom are not under control, they can affect results of the alignment. In MC simulations, they can be seen as 
distinctive patterns of deviations from real sensor positions and in differences of physical observables from truth values. In real data, one needs to estimate possible weak modes by studying their influence on various physical quantities, which have to be identified in the course of MC studies.

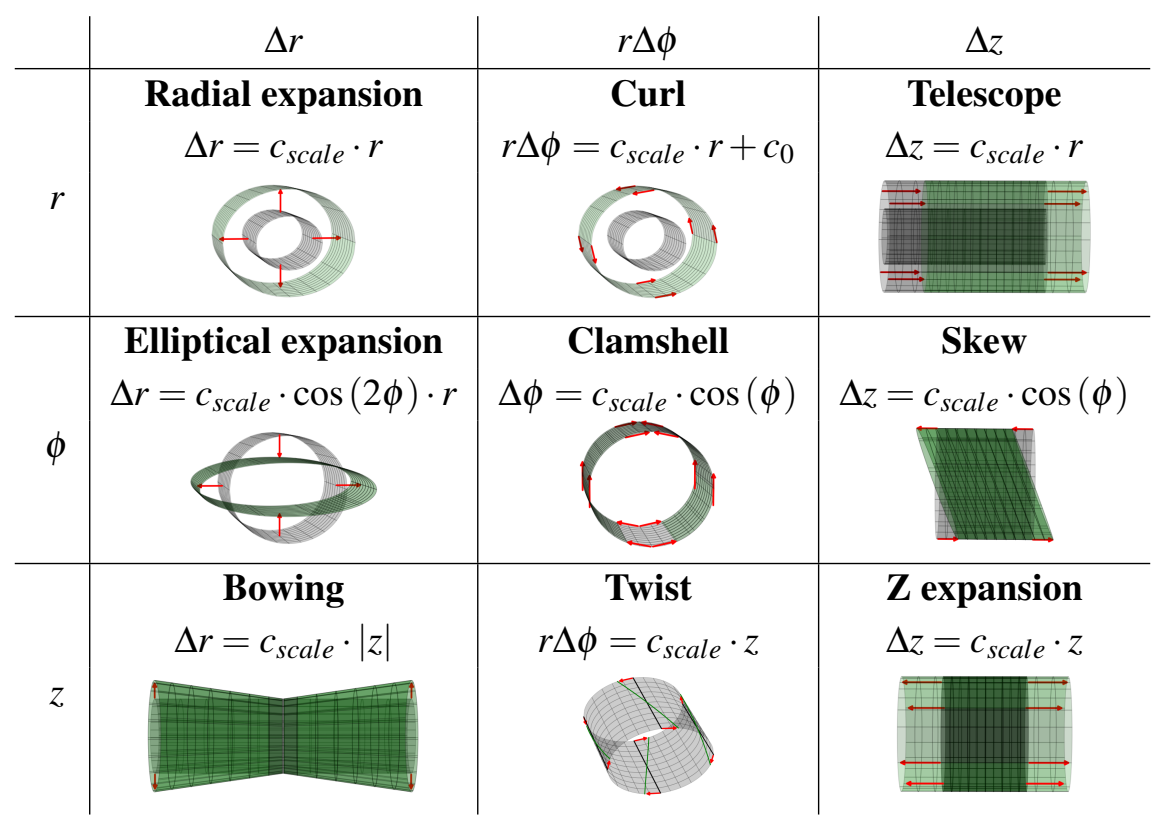

Table 2: Weak modes of the vertex detector: Coherent movements of individual sensors, which can be parametrized by displacements in global coordinates (columns) depending on the location of the sensor (rows). Each cell of the table shows the name of the weak mode (top), its parametrization (middle) and schematic illustration (bottom).

\section{Effects of misalignment on physical analysis}

Initial displacement of silicon sensors can impact physical analysis. To understanding effects of misalignment, relevant physical variables should be checked. For this analysis, we use the "golden channel" of B factories, $B^{0} \rightarrow J / \Psi\left(\rightarrow \mu^{+}+\mu^{-}\right)+K_{S}^{0}\left(\rightarrow \pi^{+}+\pi^{-}\right)$. The selected variables are positions of decay vertices, masses of primary and secondary particles, and momenta of all produced particles. The residual distributions are calculated as difference between Monte Carlo and reconstructed information.

\subsection{Random misalignment}

We can use a $\chi^{2}$ to measure the effect of misalignment on distribution of a physical parameter. This allows us to estimate the size of critical misalignment, over which the effects of misalignment become non-negligible. For small misalignment, we cannot discriminate between the effect of misalignment and statistical variability of the unperturbed distribution. We therefore define the $\chi^{2}$ of critical misalignment as the critical value of the $\chi^{2}$ test on the difference between a given and the unperturbed distribution. This also gives a logical link between the amount of data available and a minimal detected misalignment. The green bands in plots of fig. 6 are areas where misalignment cannot be detected on the background of statistical fluctuation of nominal alignment distribution. 


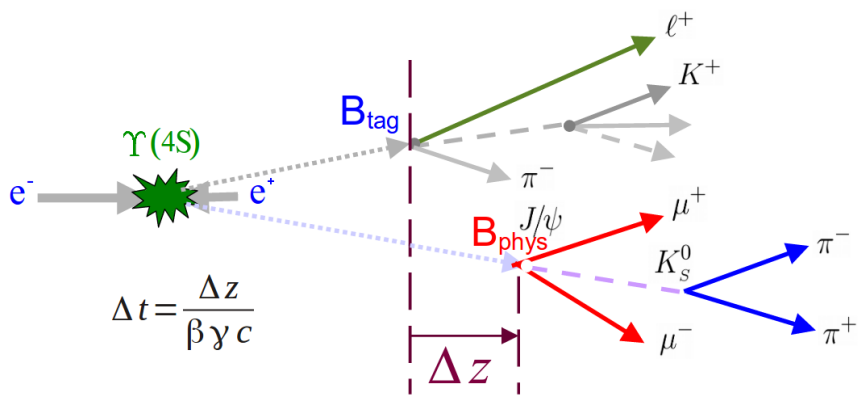

Figure 5: Schematic plot for event with decay channel $B^{0} \rightarrow J / \Psi+K_{S}^{0}$ : From collision of electron and positron at resonance $\Upsilon(4 S)$, quantum entangled pair of $\bar{B}^{0} B^{0}$ is produced. One of them is tagged and second one is reconstructed as signal. The difference between both vertices in $\mathrm{z}$-coordinate is proportional to the time difference $\Delta t$ between B decay vertices.

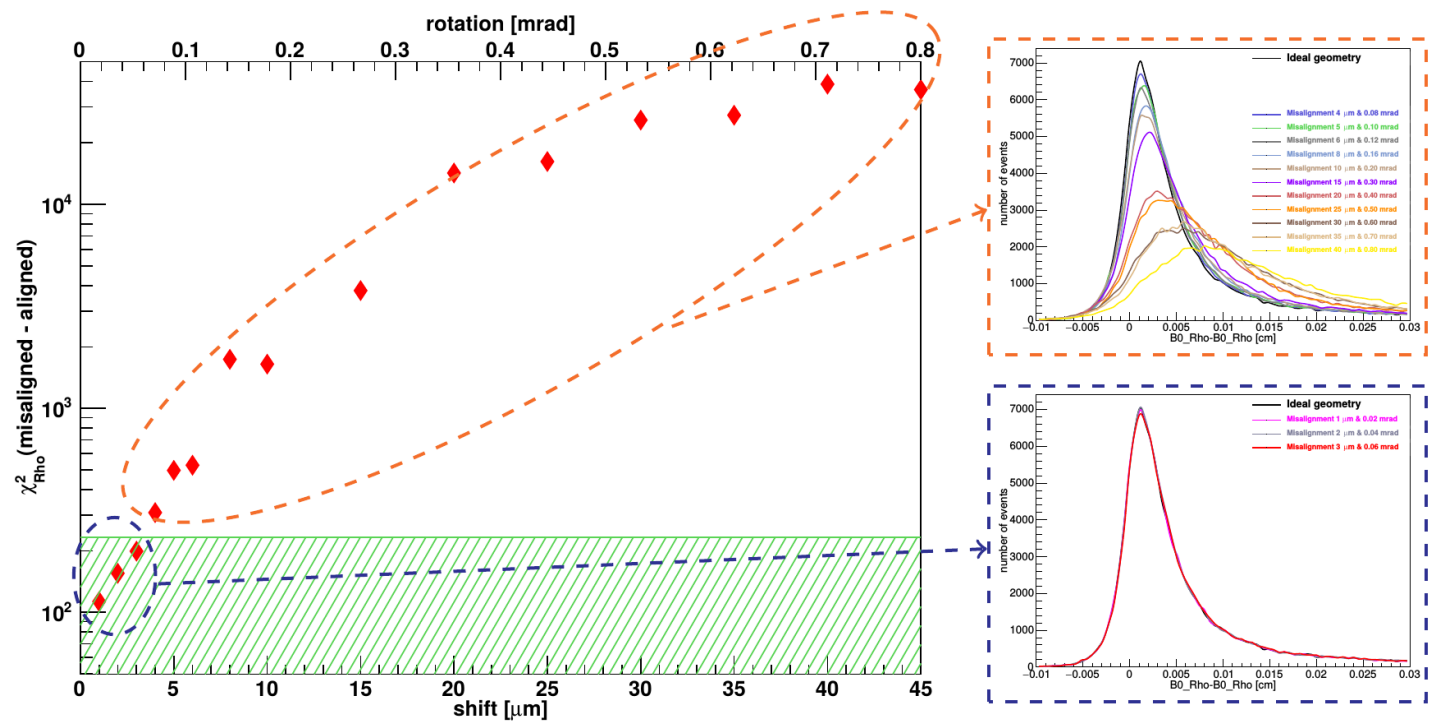

Figure 6: Effect of random misalignment on radial coordinate of $B^{0}$ vertex: The $\chi^{2}$ (left) measures statistical similarity of the distribution of the radial coordinate (right) in misaligned geometry with that of initial geometry. The green region is a $95 \%$ confidence band in which the distribution is not distinguishable from the ideal case (points correspond curves in right-bottom plot). Random misalignment with standard deviation over than $3.0 \mu \mathrm{m}$ and $0.06 \mathrm{mrad}$ are distinguishable from aligned geometry (right-top).

\subsection{Systematic misalignment}

The effects of systematics misalignment on physical analysis were measured as a difference between distributions of physical quantity for nominal (ideal) geometry and misaligned geometry. We observed several types of distribution patterns. Some distributions are shifted in positive or negative direction of $\mathrm{x}$-axis and some of them are dispersed. For radial distribution of vertex coordinates we observed similar distributions as shown in fig. 6. All observed differences are shown in tab. 3. The ampliude of the weak modes is chosen such that the maximal size of any sensors displacement is $250 \mu \mathrm{m}$. 


\begin{tabular}{|c|c|c|c|c|c|c|c|c|c|}
\hline Quantity & Radial & Elliptical & Bowing & Curl & Clamshell & Twist & Telescope & Skew & $Z$ exp. \\
\hline$B_{Z}^{0}$ residual & & & & $\mathrm{StD}$ & & StD & & StD & Shift \\
\hline$B_{\rho}^{0}$ residual & & & & $\mathrm{StD}$ & & $\mathrm{StD}$ & & & \\
\hline$J / \Psi_{Z}$ residual & & & & $\mathrm{StD}$ & & $\mathrm{StD}$ & & StD & Shift \\
\hline$J / \Psi_{\rho}$ residual & & & & $\mathrm{StD}$ & & $\mathrm{StD}$ & & & \\
\hline $\begin{array}{l}K_{S Z}^{0} \text { residual } \\
K_{S \rho}^{0} \text { residual }\end{array}$ & Shift & StD & & & & & Shift & $\mathrm{StD}$ & Shift \\
\hline$B^{0}$ mass & & & & $\mathrm{StD}$ & StD & StD & Shift & & \\
\hline$B_{P}^{0}$ residual & & & & $\mathrm{StD}$ & StD & StD & Shift & & \\
\hline$B_{P_{T}}^{0}$ residual & $\mathrm{StD}$ & & & $\mathrm{StD}$ & $\mathrm{StD}$ & StD & & & \\
\hline$B_{P_{Z}}^{0}$ residual & $\mathrm{StD}$ & & & $\mathrm{StD}$ & $\mathrm{StD}$ & $\mathrm{StD}$ & Shift & & \\
\hline$J / \Psi$ mass & & & & $\mathrm{StD}$ & StD & StD & Shift & & \\
\hline$J / \Psi_{P}$ residual & & & & $\mathrm{StD}$ & $\mathrm{StD}$ & $\mathrm{StD}$ & Shift & & \\
\hline$J / \Psi_{P_{T}}$ residual & $\mathrm{StD}$ & & & $\mathrm{StD}$ & $\mathrm{StD}$ & StD & & & \\
\hline$J / \Psi_{P_{Z}}$ residual & & & & $\mathrm{StD}$ & $\mathrm{StD}$ & StD & Shift & $\mathrm{StD}$ & \\
\hline$K_{S P}^{0}$ residual & & & & $\mathrm{StD}$ & StD & $\mathrm{StD}$ & & & \\
\hline$K_{S P_{T}}^{0}$ residual & & & & $\mathrm{StD}$ & $\mathrm{StD}$ & & & & \\
\hline$K_{S P_{Z}}^{0}$ residual & & & & $\mathrm{StD}$ & & & Shift & $\mathrm{StD}$ & \\
\hline$\mu_{P}^{ \pm}$residual & & & & Shift & $\mathrm{StD}$ & Shift & & & \\
\hline$\mu_{P_{T}}^{ \pm}$residual & $\mathrm{StD}$ & & & Shift & $\mathrm{StD}$ & Shift & & & \\
\hline$\mu_{P_{Z}}^{ \pm}$residual & StD & & & $\mathrm{StD}$ & $\mathrm{StD}$ & & Shift & StD & \\
\hline$\pi_{P}^{ \pm}$residual & & & & Shift & $\mathrm{StD}$ & $\mathrm{StD}$ & & & \\
\hline$\pi_{P_{T}}^{ \pm}$residual & & & & Shift & $\mathrm{StD}$ & & & & \\
\hline$\pi_{P_{Z}}^{ \pm}$residual & & & & $\mathrm{StD}$ & & & Shift & $\mathrm{StD}$ & \\
\hline$\Delta t$ residual & & & & & & & & & Shift \\
\hline
\end{tabular}

Table 3: Effects of weak modes on physical observables: The shown physical quantities are divided to vertex coordinates $\left(X_{Z, \rho}\right)$, momenta and its components $\left(X_{P, P_{T}, P_{Z}}\right)$ and $\Delta t$. Indices " $\rho$, $\mathrm{T}$ " label radial coordinate of a quantity and index " $Z$ " labels $\mathrm{z}$ coordinate of a quantity. The parameter " $\mathrm{X}$ " is connected with studied particle. The parameter "StD" is used for more dispersed distributions than nominal geometry distribution and "Shift" is for distributions shifted from nominal geometry distribution. Deformations in radial direction mostly do not affect physical quantities, modes in $\phi$ direction affects many of the studied quantities and deformations in $\mathrm{z}$ direction affect have quite distinc effects, depending on the parametrisation of transformation. Red physical quantities are shown in fig. 7.

\section{Alignment studies}

Our alignment study is focused on the alignment problem for the vertex detection system of Belle II. The study uses all 1272 alignment parameters (see tab. 1). To reduce the degrees of freedom, we use a set of simple constraints: We require that the sum of alignment corrections per each rigid body parameter, expressed in the global system, is zero. The alignment parameters are first calculated with nominal geometry. Three different datasets are used: single charged tracks from $\Upsilon(4 \mathrm{~S})$ resonance, cosmic muons recorded without magnetic field and vertex and IP constrained muon pairs. In this alignment study, $1 \mathrm{M}$ tracks are used in each case, but the mixture of the 

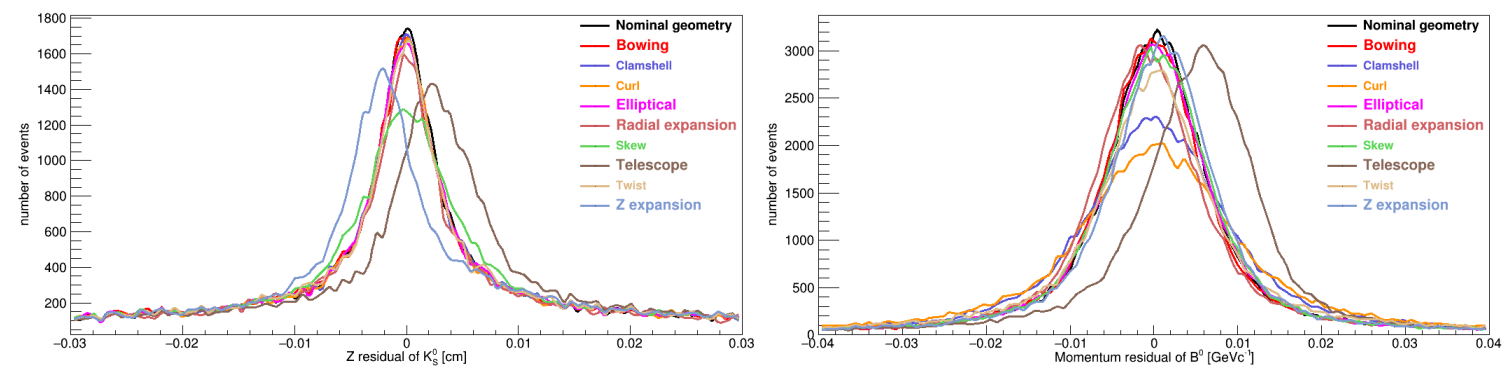

Figure 7: $Z$ residual of $K_{S}^{0}$ (left) and momentum residual of $B^{0}$ distributions (right): Distributions for all weak modes are shown.

datasets is being changed. All tracks are produced by Monte Carlo simulation in basf 2 .
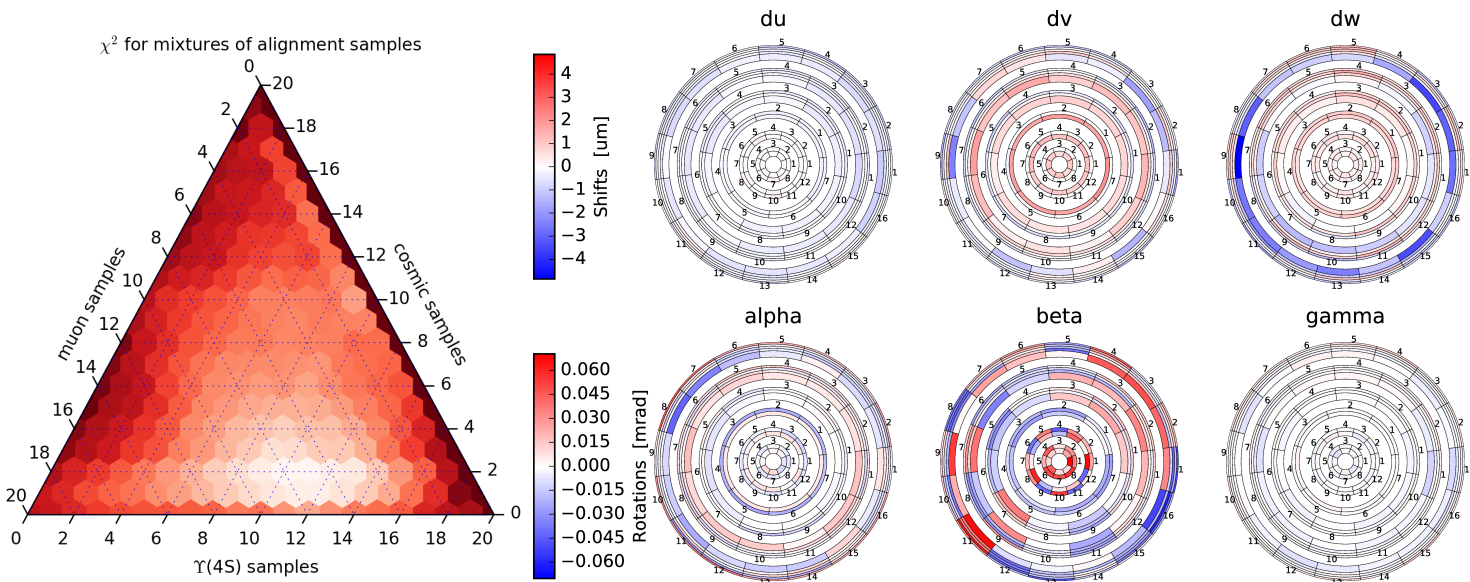

alpha

beta

gamma
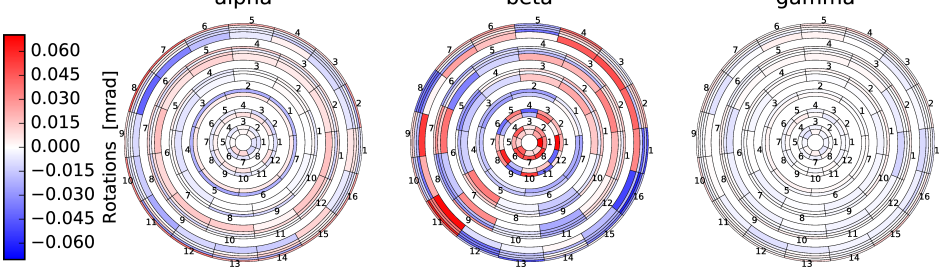

Figure 8: Finding optimal sample: The ternary plot (left) shows $\chi^{2}$ for all mixtures of the three datasets. The vertices of the triangle show "clean" datasets, the sides represent mixture of two datasets. The best choice is the mixture of $11 \Upsilon(4 S), 2$ cosmic and 7 muon samples. All alignment parameters (right) are shown for the best mixture. $\chi^{2}$ invariant modes are successfully eliminated. Largest residual misalignments is visible in the $\beta$ angle (rotation around long axis of sensors) and wedge sensors.

Alignment can be affected by $\chi^{2}$ invariant (weak) modes (described in Sec. 3.2). The $\chi^{2}$ invariant modes are not used as initial displacements of sensors, but they might emerge as a result of the alignment procedure. To $\chi^{2}$ invariant modes elimination we used a ternary plot, which maps the mixing ratios of the 3 datasets with 0.05 step. Together we have calculated 231 alignments (fig. 8). The quality of each alignment is measured by residual $\chi^{2}$, defined as a sum of parameters biases divided by their estimated errors. The "optimal sample" is a mixture with the smallest $\chi^{2}$ value of residual misalignment. The residual misalignment is defined as difference between calculated and initial geometry.

A different study of the alignment considers the convergence of alignment results and how tis depends on the initial misalignment. We generated the initial misalignment randomly. The ranges of generated misalignment parameters and calculated values of residual misalignment are shown in tab. 4. 


\begin{tabular}{cc|cccccc}
\multirow{2}{*}{ Initial } & Shifts $[\mu \mathrm{m}$ ] & 0.00 & 10.2 & 157.7 & 0.0 & 0.0 & 215.7 \\
& Rotations [mrad] & 0.000 & 0.000 & 0.000 & 0.247 & 3.040 & 3.467 \\
Residual & Shifts $[\mu \mathrm{m}$ ] & 4.62 & 4.11 & 4.06 & 4.15 & 4.09 & 4.35 \\
& Rotations [mrad] & 0.086 & 0.106 & 0.106 & 0.104 & 0.104 & 0.104
\end{tabular}

Table 4: Convergence of alignment using large scale random misalignment: The sensors are initially randomly misplaced. Shown values are the largest misplacements of sensor from nominal positions. The residual misalignment shows difference between initial and calculated position or rotation of a sensor. The residual misalignments are similar to the result of alignment procedure and it does not dependent on the initial geometry.

\section{Conclusion}

The alignment procedure of the vertex detector is an important part of the Belle II analysis and simulation framework. The procedure is developed with partial results. We implemented successfully the tools for generation of several types of misaligned geometry of the vertex detector: random and systematic misalignments. However, it is desirable to extend our method to consider also planar deformation of silicon sensors.

We should study effects of misplacements and deformations of vertex detector on physical analysis, to develop reliable online monitoring tools. The effects of misalignments on physical analysis shown are using Monte Carlo simulation of golden channel of the B factories. The random misalignment study estimates the critical misalignment for precise measurements with the vertex detector. The systematic misalignment study shows possibilities for monitoring the vertex detector misplacements using physical analysis and it shows a necessity to consider also other ways of monitoring. These results will be helpful during development of the monitoring tools. We want to use several types of collected information to evaluate detector condition dVXDuring data taking: tracking information, physical information and cosmic information.

The main part of alignment studies shows results obtained with the current version of the alignment procedure, still under development. We are able to successfully eliminate $\chi^{2}$ invariant modes using mixture of samples with different topology and our alignment procedure is stable as function of large scale random misalignment. However, the procedure should be tested using weak modes and other realistic geometries of the vertex detector. The most important question for alignment procedure will be reflection of monitoring information to alignment result and how to successfully connect prediction from monitoring to precisely determine positions of silicon sensors.

\section{Acknowledgments}

This project was supported by Charles University, project GA UK no. 404316.

\section{References}

[1] Z. Dolezal, S. Uno et al.: Technical Design Report, arxiv: 1011.0352v1,

[2] J. Kemmer and G. Lutz: Nucl. Instrum. Meth. Phys.Res. A 253, 356 (1987) 
[3] M. Friedl, T. Bergauer, A. Frankenberger, I. Gfall, C. Irmler, M. Valentan: The Silicon Vertex Detector of the Belle II Experiment [online] http://dx.doi.org/10.1016/j.phpro.2012.02.428 (cit. 2016/05/04)

[4] Belle II Collaboration: The vertex detector numbering scheme (Belle II internal document)

[5] D. Y. Kim Software and Physics Simulation at Belle II arXiv:1510.09008 [physics.ins-det]

[6] M. Stoye Calibration and Alignment of the CMS Silicon Tracking Detector, Dissertation, University of Hamburg, 2007

[7] V. Blobel, C. Kleinwort: A new method for the high-precision alignment of track detectors, arXiv:hep-ex/0208021

[8] V. Karimaki et al. Sensor Alignment by Tracks, arXiv:physics/0306034 [physics.data-an]

[9] C. Kleinwort: General Broken Lines as advanced track fitting method arXiv:1201.4320 [physics.ins-det] 